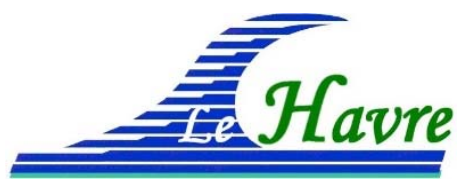

XVI ìmes Journées Nationales Génie Côtier - Génie Civil

Le Havre, 2020

DOI:10.5150/jngcgc.2020.092 (C) Editions Paralia CFL

disponible en ligne - http://www.paralia.fr - available online

\title{
Gestion des risques littoraux et trajectoires d'adaptation par méthode DAPP
}

\section{Christophe BRIERE ${ }^{1}$, Marjolijn HAASNOOT ${ }^{2,3}$}

1. Egis Ports, 15 avenue du Centre, 78280 Guyancourt, France.

christophe.briere@egis.fr

2. University Utrecht, Princetonlaan 8a, 3584 CB Utrecht, Pays-Bas.

m.haasnoot@uu.nl

3. WDM, Deltares, Boussinesqweg 1, 2629 HV Delft, Pays-Bas.

marjolijn.haasnoot@deltares.nl

\section{Résumé :}

L'approche Dynamic Adaptive Policy Pathways (DAPP) est une approche pour le soutien à la prise de décision dans un contexte de forte incertitude (Decision Making under Deep Uncertainty, DMDU). L'application de la méthode vise à une planification dynamique des mesures de gestion des risques de catastrophe, en réponse au futur tel qu'il se déroulera réellement et non pas tel qu'il aurait été prévu. Plus concrètement, plusieurs trajectoires sont généralement visualisées sur un arbre de décision, par rapport au temps ou aux conditions changeantes (comme par exemple le niveau moyen des mers). Pour cela, l'approche DAPP explore des séquences alternatives de décisions pour de multiples futurs possibles, ouvre l'espace de décision, aidant ainsi à surmonter la paralysie politique liée à l'incertitude de l'évolution des systèmes naturels, socio-économiques et institutionnels. Comme les différentes mesures de gestion des risques ont une durée de vie incertaine, une des spécificités de l'approche DAPP est de définir des points de basculement (Adaptation Tipping Points, ATP), i.e. définir lorsque les conditions de fonctionnement changent et que les objectifs de gestion des risques ne sont plus atteints. La stratégie de gestion des risques se conçoit de manière adaptative et dynamique qui comprend des actions initiales, des options à long terme, et des signaux d'adaptation, permettant d'identifier quand mettre en ouvre les options à long terme ou revoir les décisions.

\section{Mots-clés :}

Gestion des risques littoraux, Planification adaptative dynamique, Aide à la décision, Trajectoires résilientes, DAPP, Point de basculement, Signaux. 


\section{Lecture spéciale}

\section{Introduction}

De nos jours, les décideurs sont confrontés à de profondes incertitudes concernant une myriade de facteurs externes, tels que le changement climatique, la croissance démographique, les nouvelles technologies, les développements économiques et leurs impacts. De plus, non seulement les conditions environnementales, mais aussi les perspectives et les préférences de la société peuvent changer avec le temps, comme par exemple les intérêts des parties prenantes et leur évaluation des plans (OFFERMANS et al., 2011). Pour la planification de nouvelles infrastructures, où les dépenses d'investissement sont élevées et la durée de vie des actifs est longue, les décideurs doivent être convaincus que les décisions qu'ils prennent aujourd'hui continueront de s'appliquer sur le long-terme et que les actions sont conçues pour pouvoir faire face à des conditions changeantes. Or, les approches de planification traditionnelles ne permettent pas de prendre en compte ces incertitudes. Par ailleurs, la publication régulière de nouveaux scénarios climatiques signifie que les réglementations doivent être modifiées et que les plans stratégiques existants basés sur les anciens scénarios doivent tous être mis à jour. Ceci montre également la nécessité d'adopter des approches de planification moins dépendante d'un ensemble particulier de scénarios.

Cet article décrit une approche de planification dans des conditions de forte incertitude, appelée Dynamic Adaptive Policy Pathways (DAPP) (HAASNOOT, 2013; HAASNOOT et al., 2013), qui reconnaît que les décisions sont prises dans le temps en interaction dynamique avec le système concerné, et ne peuvent donc pas être considérées indépendamment les unes des autres. L'approche DAPP intègre deux approches de planification adaptative partiellement imbriquées et complémentaires: la planification adaptative dynamique (WALKER et al., 2001) et le concept de trajectoires d'adaptation (HAASNOOT et al., 2012). Egalement, le concept de points de basculement de l'adaptation ou méthode ATP (KWADIJK et al., 2010) est au cœur de l'approche DAPP. L'approche vise à recentrer le processus d'analyse des stratégies de gestion des risques sur l'identification des conditions générales dans lesquelles une stratégie ne sera plus efficace (gestion des incertitudes), plutôt que de chercher à définir ce qui peut se produire et quand (prévision), et répond donc au besoin d'être moins dépendant du scénario. En revanche, des scénarios peuvent être utilisés pour évaluer quand ces points de basculement interviendront, et quand des actions supplémentaires devront être mises en place pour continuer à atteindre les objectifs de gestion des risques. Des trajectoires commencent alors à émerger, et comme plusieurs alternatives sont possibles, plusieurs trajectoires peuvent être planifiées.

L'approche DAPP apporte donc une flexibilité dans un plan global en séquençant la mise en œuvre des actions dans le temps, de telle sorte que le système puisse être adapté aux conditions changeantes, avec des séquences alternatives spécifiées pour faire face à une gamme de conditions futures plausibles. Bien que développée à l'origine pour la mise en œuvre de trajectoires résilientes pour la gestion de l'eau, il s'agit d'une approche générique 


\section{XVIèmes Journées Nationales Génie Côtier - Génie Civil \\ Le Havre 2020}

qui peut être appliquée à d'autres problèmes de planification stratégique à long terme dans des conditions changeantes incertaines.

La section 2 présente la mise en œuvre méthodologique de l'approche DAPP en décrivant les différentes étapes successives à suivre pour l'élaboration d'un plan dynamique adaptatif, et la section 3 présente une application générique de l'approche pour le système côtier.

\section{L'approche DAPP}

Avec l'approche DAPP, un plan est conceptualisé comme une série d'actions dans le temps (trajectoires), comprenant des actions initiales et des options à long terme. Les trajectoires visent à se concentrer sur l'adaptation à des conditions changeantes (trajectoires d'adaptation), permettre des développements socio-économiques (trajectoires de développement) ou la transition vers un avenir souhaité (trajectoires de transition). La préférence pour suivre une trajectoire spécifique par rapport à d'autres dépend de l'acteur et, si la prise de décision s'appuie sur des considérations principalement économiques, dépend des compromis entre les coûts et les bénéfices des différentes trajectoires. Sur la base d'une évaluation des différentes trajectoires possibles, un plan adaptatif peut être conçu. Un suivi doit alors être mis en place pour détecter les signaux qui indiquent quand la prochaine étape, au sein d'une trajectoire, devrait être mise en œuvre, ou si une réévaluation globale du plan est nécessaire.

La figure 1 montre une schématisation de la mise en œuvre de l'approche globale DAPP Sur la base d'une analyse des problèmes pour les situations actuelles et futures, des actions sont tout d'abord identifiées pour remédier aux vulnérabilités et saisir les opportunités. Les conditions et le calendrier des points de basculement (ATP) sont ensuite évalués en fonction de leur efficacité à atteindre les résultats souhaités dans des conditions changeantes. Une fois que l'ensemble des actions est jugé adéquat, des trajectoires peuvent être conçues et évaluées. Dans la pratique, les étapes 1 à 5 de la figure 1 sont souvent d'abord exécutées qualitativement sur la base d'un jugement d'expert, suivies d'une évaluation plus détaillée de ces étapes, basée sur une modélisation. 


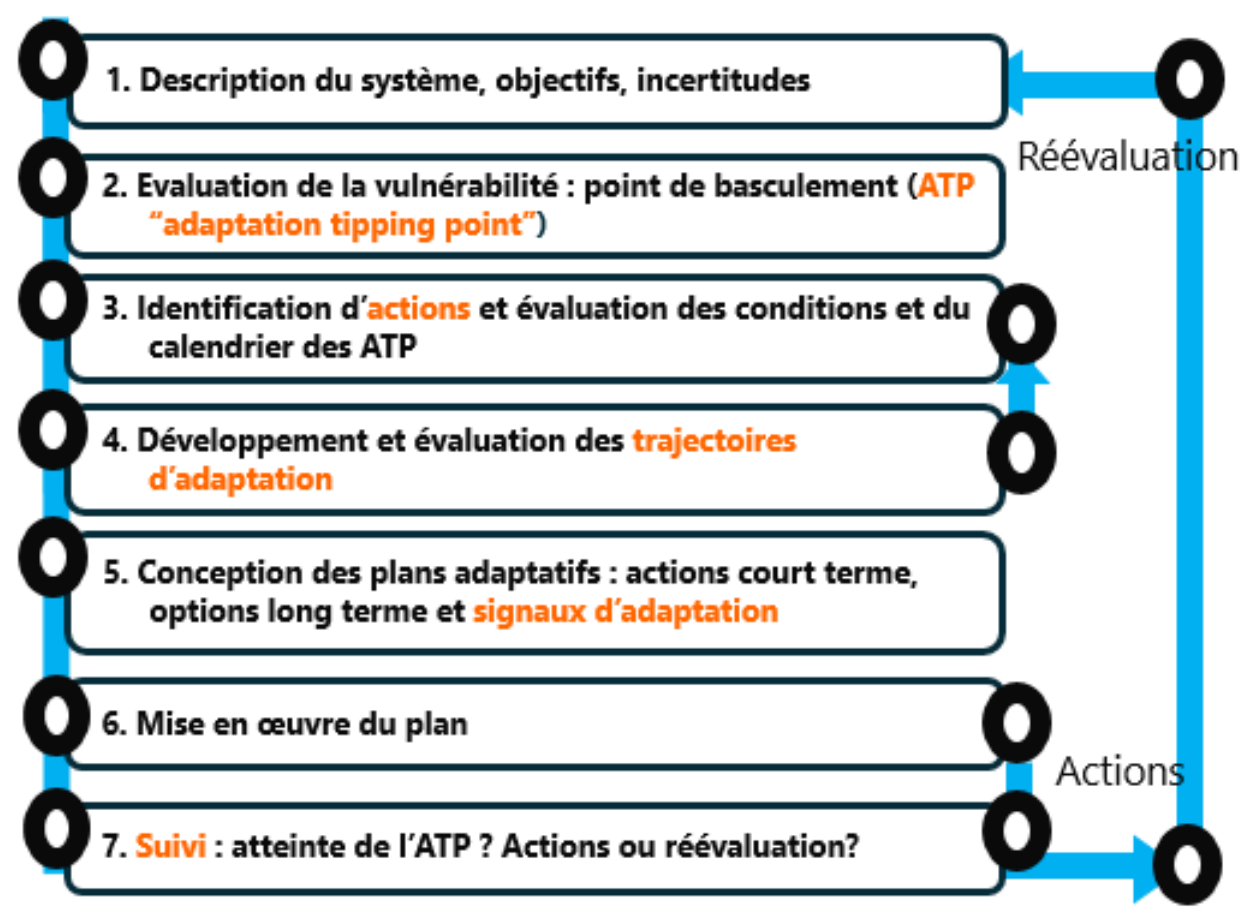

Figure 1. Schématisation de l'approche DAPP, adapté de HAASNOOT et al., (2013).

2.1 Etape 1: Cadrage des problèmes, description du système, spécification des objectifs et identification des incertitudes

La première étape consiste à décrire les caractéristiques du système, les objectifs, les contraintes dans la situation actuelle et les contraintes potentielles dans les situations futures. Le résultat de cette étape est une définition des résultats souhaités en termes de cibles et d'indicateurs. Ceux-ci sont utilisés dans les étapes suivantes pour évaluer la performance des actions et des trajectoires, et pour évaluer les conditions et le calendrier des ATP. Cette étape comprend également une spécification des principales incertitudes ou désaccords qui jouent un rôle dans la prise de décision, tels que les changements dans les forces externes, la structure du système et l'évaluation des résultats. Les incertitudes spécifiées sont utilisées pour générer un ensemble de futurs plausibles sous forme de scénarios. Ces scénarios peuvent être des scénarios statiques (décrivant un point final dans le futur) ou des scénarios transitoires (décrivant les développements au fil du temps) (HAASNOOT et al., 2015).

2.2 Etape 2: Évaluation des vulnérabilités et des opportunités du système et identification des points de basculement de l'adaptation

La deuxième étape consiste à évaluer la situation actuelle par rapport à l'ensemble des futurs plausibles, à l'aide des cibles spécifiées, afin d'identifier les conditions dans lesquelles le système ne fonctionne plus de manière acceptable (ATP). Chaque futur plausible est traité comme un "scénario de référence" en supposant qu'aucune nouvelle 


\section{XVI'èmes Journées Nationales Génie Côtier - Génie Civil \\ Le Havre 2020}

politique n'est mise en œuvre, et couvre les incertitudes identifiées à l'étape 1. Les opportunités et les vulnérabilités doivent être prises en compte, la première catégorie étant constituée des développements qui peuvent aider à atteindre les objectifs définis, et la seconde étant constituée des développements qui peuvent empêcher d'atteindre les objectifs.

Différentes approches peuvent être utilisées pour identifier les ATP. Celles-ci incluent des évaluations de vulnérabilité "ascendantes" qui établissent des seuils de résultats inacceptables avant d'évaluer la temporalité des points de basculement à l'aide de scénarios, ainsi que des approches "descendantes" qui utilisent des analyses de scénarios traditionnelles pour déterminer la portée et le moment de ces points. Les approches "ascendantes" peuvent s'appuyer sur des évaluations basées sur des modèles (par exemple des tests de résistance, des analyses de sensibilité, etc.) pour établir les conditions de défaillance (seuils), ou celles-ci peuvent être spécifiées via un jugement d'expert ou une consultation des parties prenantes. Les approches "descendantes" dépendent largement des évaluations basées sur des modèles et peuvent utiliser soit plusieurs scénarios statiques ou des scénarios transitoires (voir HAASNOOT et al., 2012) pour représenter la variété des incertitudes et leur évolution dans le temps. Les résultats de cette analyse révèlent si et quand des actions sont nécessaires pour atteindre les résultats souhaités. Une telle analyse peut être utile en cas d'incertitudes multiples et corrélées pouvant être présentes dans des scénarios.

2.3 Etape 3: Identification des actions contingentes et évaluation de leurs points de basculement (ATP) et de leur calendrier

Sur la base de l'analyse des problèmes des étapes 1 et 2, des actions alternatives sont identifiées pour atténuer les vulnérabilités et saisir les opportunités. Comme à l'étape 2 , les conditions et le calendrier des ATP pour chacune des actions identifiées sont évalués en fonction de leur efficacité à atteindre les résultats souhaités dans des conditions changeantes, en utilisant la même approche qu'auparavant. Les actions inefficaces sont éliminées (WALKER, 1988), et seules les actions prometteuses sont utilisées dans les étapes suivantes comme blocs de construction pour l'assemblage des trajectoires d'adaptation.

\subsection{Etape 4: Conception et évaluation des trajectoires}

Une fois que l'ensemble des actions est jugé adéquat, des trajectoires peuvent être conçues et évaluées. Les alternatives peuvent être non seulement des actions uniques, mais peuvent également inclure des groupes d'actions qui peuvent être exécutées simultanément. Le résultat est une carte des trajectoires (figure 2), qui résume toutes les actions politiques possibles et les trajectoires logiques dans lesquelles des actions sont combinées pour atteindre les objectifs spécifiés dans des conditions changeantes. Ainsi, les ATP établis à l'étape 3 peuvent être utilisés pour explorer toutes les trajectoires 


\section{Lecture spéciale}

possibles s'appuyant sur toutes les actions alternatives disponibles. La carte peut montrer différents axes correspondant à plusieurs conditions changeantes. Il est alors intuitivement possible d'identifier les opportunités, les actions sans regret, les blocages et le calendrier des actions, afin de soutenir la prise de décision dans un environnement en mutation. Certaines séquences d'actions peuvent être impossibles, indésirables ou moins probables (par exemple, lorsqu'elles impliquent un transfert très coûteux).

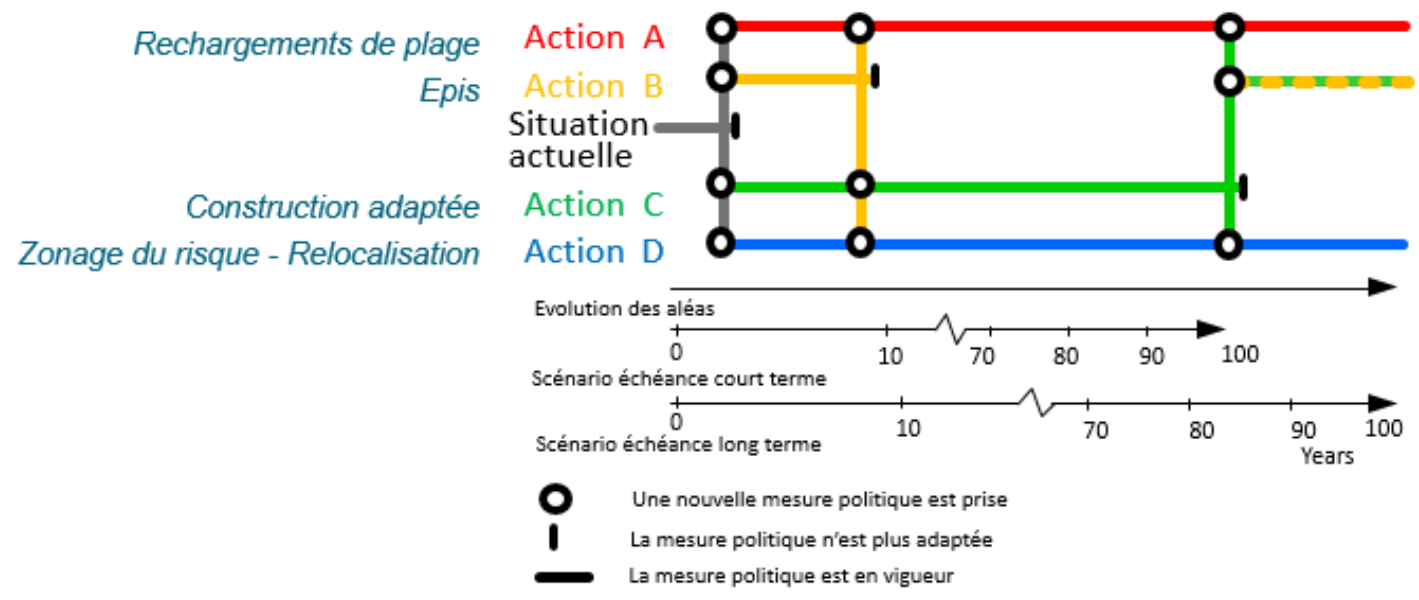

Figure 2. Exemple de carte des trajectoires, adapté de HAASNOOT et al., (2018).

Les trajectoires peuvent être développées et explorées directement à l'aide de modèles (par exemple des modèles d'optimisation multi-objectifs ou basés sur des agents (KWAKKEL et al., 2015)), de jeux sérieux (LAWRENCE \& HAASNOOT, 2017), ou plus qualitativement pendant des discussions de groupe avec les parties prenantes (par exemple, CAMPOS et al., 2016).

Dans les discussions de groupe, les parties prenantes identifient les actions initiales et les options à long terme sur la base de leur jugement d'expert, éventuellement étayées par des résultats de modélisation, puis tracent des trajectoires. L'élaboration de "scénarios", qui prennent en considération les conditions socio-économiques et environnementales et spécifient les déclencheurs d'adaptation appropriés, est une autre façon de développer des trajectoires directement avec les parties prenantes.

Dans la pratique, les points de basculement et les trajectoires sont souvent d'abord définis et développés qualitativement selon le jugement des experts avant de réaliser des analyses quantitatives plus élaborées basées sur des modélisations.

Chaque trajectoire est ensuite évaluée (par exemple à l'aide de tableaux de bord, au travers d'une analyse coûts-bénéfices, d'une analyse multicritère ou d'une évaluation de l'efficacité) sur sa performance, ainsi que sur d'autres critères fondamentaux, tels que l'urgence des actions, la gravité des impacts, l'incertitude, la dépendance des décisions à la trajectoire suivie et le désir de garder ouvertes les options futures. Les coûts et les 


\section{XVI'̀mes Journées Nationales Génie Côtier - Génie Civil \\ Le Havre 2020}

avantages peuvent changer au fil du temps, ce qui peut être pris en compte en utilisant des tableaux de bord distincts pour différentes périodes.

Sur la base de cette évaluation qui illustre les compromis entre les différentes trajectoires, un nombre gérable de trajectoires prometteuses et préférables peuvent être identifiées et soumises à une évaluation économique plus poussée, qui doit non seulement prendre en compte les coûts initiaux et récurrents de toute action, mais également les coûts associés au transfert d'une action à une autre (par exemple pour les modifications ou les impacts dus à la relocalisation, la suppression ou les ajustements de l'action précédente).

\subsection{Etape 5: Conception de la stratégie adaptative}

La cinquième étape consiste à identifier les actions initiales et les options à long terme pour un ensemble de trajectoires préférées qui sont sélectionnées sur la base de compromis. Ceci inclut la spécification d'actions correctives, défensives et de capitalisation (KWAKKEL et al., 2010) permettant de rester sur la bonne trajectoire, et un ensemble d'actions préparatoires permettant de garder ouvertes aussi longtemps que possible les options à long terme. Une telle analyse peut impliquer l'identification des conditions institutionnelles et socioculturelles qui peuvent mettre en évidence des trajectoires privilégiées, comme l'ont fait VAN DER BRUGGE \& ROOSJEN (2016).

Le plan adaptatif nécessite également un système de surveillance associé, permettant de détecter des signaux qui indiquent la nécessité de mettre en œuvre les prochaines actions. Idéalement, un système de surveillance détecte des signaux suffisamment avant qu'une décision ne soit prise pour mettre en œuvre des actions. La spécification des paramètres de suivi et la méthode d'analyse des informations sont importantes pour assurer une préparation suffisante pour la mise en place des actions suivantes. Le signal peut être différent de l'ATP associé, qui est lié aux objectifs (impacts). Les signaux peuvent être donc plutôt liés aux forces motrices, telles que les tendances et les événements dans l'environnement physique, les impacts anthropiques sur le système, les développements technologiques ou les changements dans les valeurs et les perspectives de la société (HAASNOOT et al., 2018).

La prise en compte des coûts de transfert dans l'évaluation économique des trajectoires est un autre aspect important de la mise en place de la stratégie adaptive. Les études d'évaluation économique standard sont orientées vers la caractérisation des retours sur investissement à court terme en utilisant un horizon temporel court. Ainsi, ces évaluations présentent de sérieuses limites dans l'évaluation de l'impact économique des décisions actuelles pour les infrastructures à long terme et dans l'évaluation de leurs performances dans le temps dans un contexte d'incertitude importante (OCDE, 2013). Or, lorsque les coûts de transfert sont pris en compte, les coûts économiques associés à certaines trajectoires peuvent augmenter de manière significative, de sorte que ces investissements deviennent moins attractifs. 


\section{Lecture spéciale}

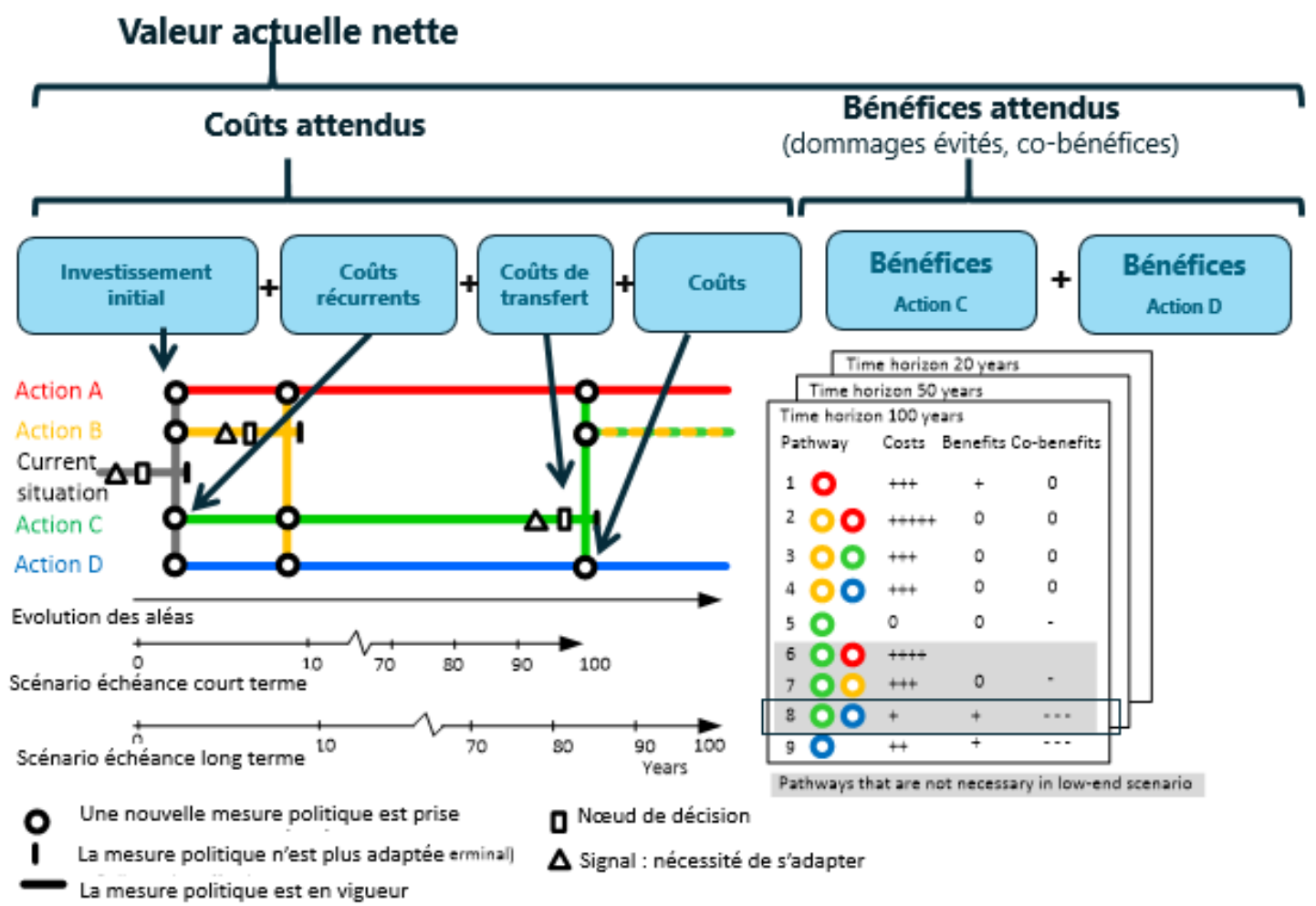

Figure 3. Carte des trajectoires et tableau de bord présentant les coûts et les avantages des trajectoires présentées sur la carte, adapté de HAASNOOT et al., (2019a).

Après avoir produit la feuille de route, un plan d'action est élaboré, précisant les actions à entreprendre immédiatement (actions initiales), ainsi qu'un aperçu des options à plus long terme, des mesures nécessaires pour les maintenir ouvertes, des développements à suivre, et des conditions dans lesquelles les prochaines actions devraient être menées. Le plan doit essentiellement résumer les résultats de toutes les étapes précédentes, y compris les objectifs, problèmes, trajectoires potentielles et préférées, et système de suivi, (figure3).

\section{6 Étape 6: mettre en œuvre la stratégie et étape 7: faire le suivi de la stratégie}

Enfin, les actions initiales et les actions nécessaires pour maintenir ouvertes les options à long terme sont mises en œuvre et le système de suivi est mis en place. Après la mise en œuvre des actions initiales, l'activation des autres actions est suspendue jusqu'à ce qu'un signal soit donné.

\section{Application générique pour le système côtier}

Coordonné par l'Université Polytechnique de Catalogne (UPC), avec la Fondation Deltares entre autres comme partenaire, le projet RISES-AM (FP7 Collaborative Project, 2013-2016) a eu pour objectif d'évaluer les impacts de l'élévation future du niveau de la mer et l'efficacité de différentes options et stratégies d'adaptation côtière. Le projet a 


\section{XVI'̀mes Journées Nationales Génie Côtier - Génie Civil \\ Le Havre 2020}

également examiné les obstacles à la mise en œuvre de l'adaptation à l'échelle locale, régionale et mondiale, pour différentes trajectoires représentatives d'évolution des concentrations (RCPs) et des trajectoires socio-économiques communes (SSPs), et tenant compte de scénarios en limite supérieure non inclus dans les rapports du GIEC.

Sur la base du projet RISES-AM, six archétypes côtiers ont été identifiés. Ces archétypes côtiers seront impactés par l'élévation du niveau de la mer, et comprennent les littoraux de basse altitude, les deltas et les estuaires. Les impacts, en termes de victimes et de dommages économiques, environnementaux et sociaux, seront cependant différents selon que les zones concernées soient urbanisées (industrielles) ou rurales. Par conséquent, les stratégies d'adaptation (figure 4) pour chacune de ces zones côtières à risque seront également différentes.

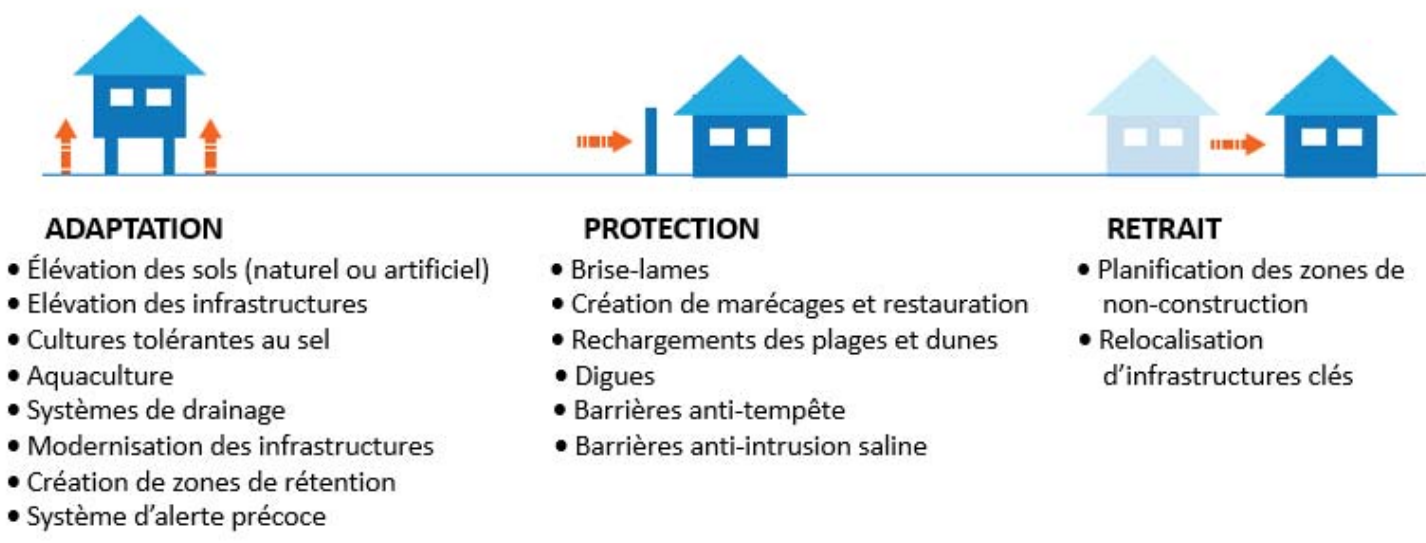

Figure 4. Stratégies et options d'adaptation possible en zone côtière.

Pour les six archétypes côtiers définis, des trajectoires génériques d'adaptation ont été développées (HAASNOOT et al., 2019b; BROWN et al., 2016; voir exemple en figure 5). Ces cartes génériques peuvent servir d'inspiration pour développer des trajectoires d'adaptation plus spécifiques pour toute zone côtière appartenant aux archétypes. Néanmoins, pour y parvenir, il est nécessaire d'identifier les objectifs de politique locale et d'évaluer les mesures d'adaptation pertinentes et leurs points de basculement.

Les études de cas RISES-AM ont clairement montré que les barrières financières et institutionnelles constituent le plus grand défi de l'adaptation côtière. Sur le plan financier, l'adaptation nécessite souvent de gros investissements initiaux difficiles à mobiliser, même si l'investissement est rentable à long terme. D'un point de vue institutionnel, il n'y a pas de mécanismes appropriés pour faire face aux différents enjeux. Le projet RISESAM a conclu que les autorités locales et nationales devraient être encouragées à développer des méthodes de planification adaptative pour pouvoir gérer les incertitudes existantes dans les impacts associés à l'élévation du niveau de la mer. Les trajectoires 


\section{Lecture spéciale}

d'adaptation côtière peuvent être ensuite utilisées pour centrer les décisions sur un certain nombre de stratégies et d'options crédibles d'adaptation côtière.

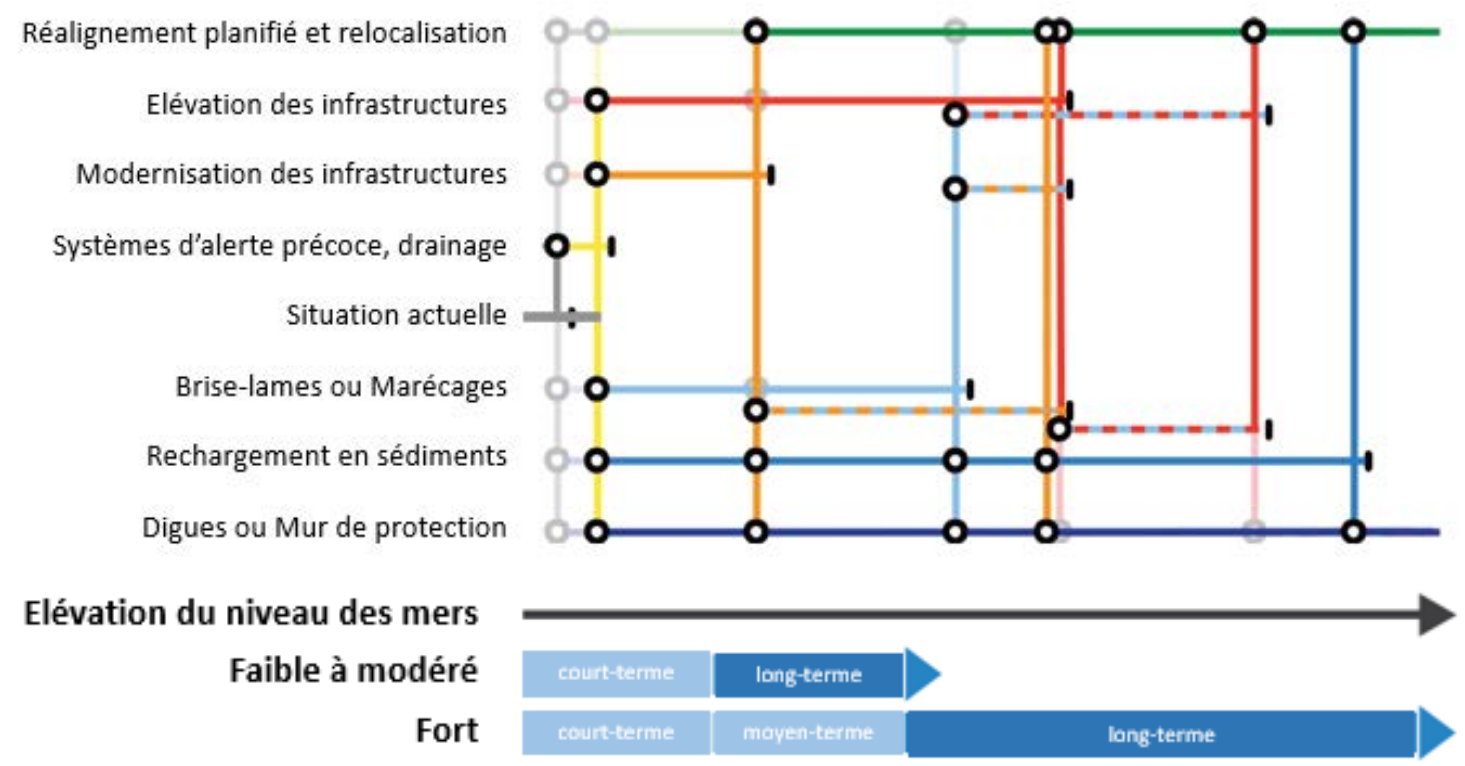

Figure 5. Carte DAPP, avec options d'adaptation en colonne de gauche, présentée pour une côte urbanisée ouverte avec plage et / ou dunes de sable. Les trajectoires les moins susceptibles de se produire sont indiquées en semi-transparence.

\section{Conclusions}

Les approches de planification adaptative sont particulièrement utiles pour aménager les systèmes physiques complexes, où il existe de grandes incertitudes et de multiples décisions de gestion à prendre sur de longues périodes et où la dépendance de ces décisions sur les trajectoires d'actions est forte. Dans de tels systèmes, les décisions d'investissement à long terme sont particulièrement exposées aux facteurs de changement. Sans tenir compte de la dépendance à long terme, des actions inadaptées peuvent en résulter, laissant les actifs bloqués bien avant que leur durée de vie nominale ne soit atteinte. Les trajectoires d'adaptation peuvent aider à limiter ces risques en aidant à identifier la séquence d'action la plus appropriée avec une flexibilité d'adaptation aux conditions changeantes.

L'approche DAPP vise à faciliter la prise de décision en offrant des visualisations intuitivement compréhensibles des différentes options politiques, et en stimulant les planificateurs préparant les décisions à reconnaître les incertitudes et à les inclure explicitement dans leurs plans - pour définir les actions à mener dès maintenant et les options ouvertes à long terme. L'identification des points de basculement de l'adaptation et des opportunités, et le développement de trajectoires, incitent les planificateurs, les décideurs et les parties prenantes à discuter et à considérer les limites possibles de 


\section{XVİ̀mes Journées Nationales Génie Côtier - Génie Civil \\ Le Havre 2020}

l'adaptation du fait de conditions changeantes incertaines, ainsi que les impacts potentiels à plus long terme pour les générations futures. Ils sont encouragés à penser de manière plus large que par la mise en place d'actions traditionnelles, et en particulier à envisager les possibilités d'actions de transformation plus importantes pour faire face à de grands changements dans le système qui dépassent les limites des pratiques actuelles. L'espace de décision peut ainsi être élargi et les difficultés futures anticipées.

\section{Références bibliographiques}

BROWN S., HAASNOOT M., BUCX T., VAN DE BRUGGE R., WADEY M., NICHOLLS R.J., et al. (2016). Report on generic adaptation roadmap with reduced impact uncertainty (per selected case studies and with an across scales component). RISES-AM EU Research project. Deliverable 4.2.

CAMPOS I.S., ALVES F., DINAS J., TRUININGER M., VIZINHO A., PENHALOPES G. (2016). Climate adaptation, transitions, and socially innovative actionresearch approaches. Ecology and Society, Vol. 21(1):13. http://dx.doi.org/10.5751/ES-08059210113

HAASNOOT M. (2013). Anticipating change - Sustainable water policy pathways for an uncertain future. Ph.D. dissertation, Twente University, Enschede, Netherlands. https://doi.org/10.3990/1.9789036535595

HAASNOOT M., KWAKKEL J.H., WALKER W.E., TER MAAT J. (2013). Dynamic adaptive policy pathways: A method for crafting robust decisions for a deeply uncertain world. Global Environmental Change, Vol. 23(2), pp 485-498. https://doi.org/10.1016/j.gloenvcha.2012.12.006

HAASNOOT M., MIDDELKOOP H., OFFERMANS A., VAN BEEK E., VAN DEURSEN W.P.A. (2012). Exploring pathways for sustainable water management in river deltas in a changing environment. Climatic Change, Vol. 115(3-4), pp 795-819. https://doi.org/10.1007/s10584-012-0444-2

HAASNOOT M., SCHELLEKENS J., BEERSMA J.J., MIDDELKOOP H., KWADIJK J.C.J. (2015). Transient scenarios for robust climate change adaptation illustrated for water management in the Netherlands. Environmental Research Letters, Volume 10, Number 10, 17p. https://doi.org/10.1088/1748-9326/10/10/105008

HAASNOOT M., VAN AALST M., ROZENBERG J., DOMINIQUE K., MATTHEWS J., BOUWER L., KIND J., LEROY N. (2019a). Investments under non-stationarity: economic evaluation of adaptation pathways. Climatic Change 161, pp 451-463, https://doi.org/10.1007/s10584-019-02409-6

HAASNOOT M., BROWN S., SCUSSOLINI P., JIMENEZ J.A., VAFEIDIS A.T., NICHOLLS R.J. (2019b). Generic adaptation pathways for coastal archetypes under uncertain sea-level rise. Environ. Res. Commun. 1 (2019) 071006, Vol. 1(7), 12 p. https://doi.org/10.1088/2515-7620/ab1871 


\section{Lecture spéciale}

HAASNOOT M., VAN KLOOSTER S., VAN'T ALPHEN J. (2018). Designing a monitoring system to detect signals to adapt to uncertain climate change. Global Environmental Change, Vol. 52, pp 273-285. https://doi.org/10.1016/j.gloenvcha.2018.08.003

KWADIJK J.C.J., HAASNOOT M., MULDER J.P.M., HOOGVLIET M.M.C., JEUKEN A.B.M., VAN DER KROGT R.A.A., VAN OOSTROM N.G.C., SCHELFHOUT H.A., VAN VELZEN E.H., VAN WAVEREN H., DE WIT M.J.M. (2010). Using adaptation tipping points to prepare for climate change and sea level rise: A case study in the Netherlands. Wiley Interdisciplinary Review Climate Change, Vol. 1(5), pp 729-740. https://doi.org/10.1002/wcc.64

KWAKKEL J.H., HAASNOOT M., WALKER W.E. (2015). Developing dynamic adaptive policy pathways: A computer-assisted approach for developing adaptive strategies for a deeply uncertain world. Climatic Change, Vol. 132, pp 373-386. https://doi.org/10.1007/s10584-014-1210-4

KWAKKEL J.H., WALKER W.E., MARCHAU V.A.W.J. (2010). Adaptive airport strategic planning. European Journal of Transport and Infrastructure Research, Vol. 10(3), pp 249-273. https://doi.org/10.18757/ejtir.2010.10.3.2891

LAWRENCE J., HAASNOOT M. (2017). What it took to catalyse uptake of dynamic adaptive pathways planning to address climate change uncertainty. Environmental Science \& Policy, Vol. 68, pp 47-57. https://doi.org/10.1016/j.envsci.2016.12.003

OCDE. (2013).Water and climate change adaptation: Policies to navigate uncharted waters. Paris: OECD Publishing.

OFFERMANS A., HAASNOOT M., VALKERING P. (2011). A method to explore social response for sustainable water management strategies under changing conditions. Sustainable Development, Vol. 19, pp 312-324. https://doi.org/10.1002/sd.439

VAN DER BRUGGE R., ROOSJEN R. (2016). An institutional and sociocultural perspective on the adaptation pathways approach. Journal of Water and Climate Change, 6(4), pp 743-758. https://doi.org/10.2166/wcc.2015.001

WALKER W.E. (1988). Generating and screening alternatives, Chapter 6, in H. J. Miser \& E. S. Quade (Eds.), Handbook of systems analysis: Craft issues and procedural choices. North Holland, NY.

WALKER W.E., RAHMAN S.A., CAVE J. (2001). Adaptive policies, policy analysis, and policymaking. European Journal of Operational Research, Vol. 128(2), pp 282-289. https://doi.org/10.1016/S0377-2217(00)00071-0 